\title{
Avaliação dos níveis de expectativa e motivação de estudantes de graduação do ensino presencial através da Mineração de dados
}

\begin{abstract}
This article presents a practical study to evaluate the levels of expectation and motivation of undergraduate students in face-to-face to their current semester and to show how their abilities can influence this result. This research was done through the use of tasks of data mining and visualization of information with the cloud tag technique. The results allowed to verify the levels of motivation of the students with their respective graduation period. Through the analysis of the data it was verified that the motivation is still strongly linked to the methodology of teaching used in the classroom by the faculty and that previous knowledge related to the undergraduate course is not necessarily a preponderant factor to the high motivational state.
\end{abstract}

Resumo. Este artigo apresenta um estudo prático para avaliação dos níveis de expectativa e motivação de estudantes de graduação do ensino presencial em relação ao seu atual semestre e mostra de que maneira as suas habilidades podem influenciar neste resultado. Essa pesquisa deu-se por meio da utilização de tarefas de mineração de dados $e$ visualização de informações com a técnica de nuvens de tags. Os resultados permitiram verificar os níveis de motivação dos alunos com seu respectivo período de graduação. Através da análise dos dados foi verificado que a motivação ainda está fortemente ligada à metodologia de ensino empregada em sala de aula pelo corpo docente e que conhecimentos prévios relacionados ao curso de graduação não são necessariamente um fator preponderante ao alto estado motivacional.

\section{Introdução}

No Brasil, a educação superior é ofertada em três modalidades: Presencial, em que todas as disciplinas são ofertadas em sala de aula física; Educação a Distância (EaD) pela qual os componentes curriculares são ministrados online, salvo as atividades obrigatoriamente consumadas presencialmente; Semipresencial, na qual uma parcela das disciplinas são oferecidas de modo presencial e outras a distância.

Dentro do Ensino Superior Presencial existem as Universidades, as quais são protagonistas no ensino-aprendizagem e têm enfrentado obstáculos, tais como a conquista de novos públicos, por meio da reavaliação do seu papel enquanto ambiente formador de pessoas com pensamentos livres. A função encorajadora de estudante face ao conhecimento e a relação com os métodos de formação de profissionais, que impulsionam a procura de novas práticas metodológicas [GOUVEIA, 2005]. 
VII Congresso Brasileiro de Informática na Educação (CBIE 2018)

Anais dos Workshops do VII Congresso Brasileiro de Informática na Educação (WCBIE 2018)

Outro protagonista no processo de ensino-aprendizagem são os alunos, pois cada estudante, seja de cursos diferentes ou de uma mesma graduação, possui particularidades, sendo elas válidas para que se possa conhecer seus interesses, suas motivações e suas habilidades logo no início do curso ou de um determinado período, a fim de que se consiga planejar melhor uma determinada aula ou curso e, com tal medida, promover ajustes que minimizem a evasão e aumente os índices ou níveis de aprendizagem.

Dentre os fatores, cujas ausências culminam no processo de evasão, estão a motivação, encarada como o engajamento do estudante com as atividades acadêmicas sem a interferência de qualquer fator, como recompensas, pressões entre outros; e a expectativa, que são predições, que os estudantes fazem, não apenas em relação ao seu desempenho acadêmico ou formação qualificadora, mas também em seu convívio social dentro do ambiente acadêmico.

Neste contexto, é importante a realização de pesquisas que possam mostrar padrões presentes em estudantes universitários do ensino presencial, no que se refere as seguintes variáveis: Expectativas e motivações e de que maneira suas habilidades técnicas podem influenciar nessas mudanças. Ou seja, como estabelecer níveis de motivação entre estudantes de graduação do ensino presencial tendo como base suas expectativas e habilidades?

O presente trabalho teve como objetivo analisar a viabilidade da concatenação de tarefas típicas da Mineração de dados, como associação e classificação, na condição de suporte para a construção de parâmetros relacionados à motivação e desapontamento de estudantes graduandos na modalidade de ensino presencial.

Este artigo está estruturado da seguinte forma: Na três secções seguintes é apresentada a fundamentação teórica juntamente com os trabalhos relacionados, já na quarta secção é apresentada a metodologia empregada para atingir os resultados, em seguida, é exposto os resultados encontrados e por fim, a última seção contempla a conclusão dessa pesquisa.

\section{Mineração de Dados}

Para Sumathi e Sivanandam (2006) a mineração de dados é uma tecnologia recente e promissora. Pode ser compreendida como uma metodologia empregada no descobrimento de conjuntos significativos de dados em um reposit 'orio, que emprega técnicas estat 'isticase de visualização de dados. Essa tecnologia deve permitir a exploração de tendências e padrões preditivos em dados, a fundamentação e o teste de hipóteses e a geração de informações instigantes. Ainda de acordo com os autores, dados podem ser entendidos como qualquer $\mathrm{n}$ umero ou texto que pode ser processado por um computador.

\subsection{Data Mining na Educação}

Historicamente, a utilização de algoritmos de Data mining na educação é uma via relativamente nova. Embora muitos pesquisadores armazenem e explorem dados de softwares educacionais há um bom tempo, apenas recentemente este caminho foi estabelecido como um campo próprio. Scheuer e McLaren (2012), a Mineração de Dados Educacionais concentra-se em elaborar, estudar e executar procedimentos computadorizados para descobrir padrões em grandes conjuntos de dados educacionais que, de outra maneira, seriam difíceis ou improváveis de explorar devido ao vasto volume de dados que existem. 
VII Congresso Brasileiro de Informática na Educação (CBIE 2018)

Anais dos Workshops do VII Congresso Brasileiro de Informática na Educação (WCBIE 2018)

\section{Questionário como forma de coleta de dados}

A investigação de dados para exploração quantitativa por meio de questionários exige cuidado específico. Recomenda-se declarar que não é apenas necessário reunir respostas sobre questões relevantes, mas conseguir analisá-las matematicamente para corroboração dos resultados. A ajuda estatística numa pesquisa quantitativa assiste o pesquisador que não conhece as condições básicas a serem atendidas em pesquisas de campo (Manzato e Santos, 2012).

De acordo com Costa (2016) uma das ferramentas mais utilizadas para coleta de dados'e o questionário, entre as suas vantagens, são listadas as seguintes:

- Obtém grande número de dados;

- Compreende um grande número de participantes simultaneamente;

- Economiza pessoal, no que se refere ao treinamento para coleta de dados em campo;

- Obtém respostas mais rápidas e exatas;

- Oferece liberdade de respostas, ou seja, permite o anonimato;

- Permite flexibilidade de horário as pessoas para responderem aos questionamentos.

\section{Trabalhos Relacionados}

O trabalho de Cavalcanti et al. (2014) expõe um método prático para análise dos níveis de motivação de alunos na modalidade EAD (Educação a Distância). Essa indicação deu-se com base na combinação de algoritmos de mineração de dados educacionais e de visualização de palavras com a técnica de nuvens de tags. Os resultados encontrados pelos autores possibilitaram a verificação dos níveis de motivação. Com amostras específicas verificou-se que a motivação é boa, contrariando algumas referências que revelam dificuldades na adequação à EAD e altos índices de evasão.

Já na obra de Ramos et al. (2014) é apresentado um estudo que mostra traços da variação dos níveis de expectativas e de habilidades de estudantes em um curso de graduação do ensino à distância. Com base na aplicação de questionários no início e no final de uma disciplina e subsequentes tratamentos dos dados coletados, os resultados indicam que as expectativas dos alunos ao iniciarem um curso mudam consideravelmente ao longo de uma disciplina. Outro elemento relevante foi a compreensão de alterações nas habilidades obtidas, já que, de acordo com os autores do estudo, isto pode ser um indicativo de acréscimo na autoconfiança dos alunos para prosseguirem com os seus estudos.

No trabalho de Santos et al. (2016), os autores apresentam um modelo computacional conceitual sobre a aplicação de técnicas de Mineração de Dados com foco na Análise de Sentimentos de forma a despertar a percepção dos alunos acerca das práticas docente, além disso, buscam compreender as expectativas e limitações dos estudantes e atribui ao professor juntamente com a instituição de ensino o papel de modelar o que é possível para garantir o aprendizado dos discentes. 
VII Congresso Brasileiro de Informática na Educação (CBIE 2018)

Anais dos Workshops do VII Congresso Brasileiro de Informática na Educação (WCBIE 2018)

Nesta perspectiva, o presente artigo se diferencia dos demais por utilizar a linguagem de programação Python em tarefa de Mineração de dados e a exposição dessa ferramenta, amplamente difundida em curso de computação, porém pouco explorada para esse fim para análise dos níveis motivação e expectativa de estudantes de graduação da modalidade presencial, público este não abordado nas obras expostas.

\section{5 . Procedimentos Metodológicos}

\subsection{Participantes}

Participaram da presente pesquisa um total de 203 estudantes de 28 cursos de graduação, como mostra a Figura 1, do ensino presencial de instituições do ensino superior tanto públicas quanto privadas. Para a escolha dos candidatos da pesquisa, foi levado em consideração se eram ou não estudantes de graduação. Quem era estudante graduação a sua resposta foi contabilizada e processada. $\mathrm{O}$ mesmo não aconteceu para os não estudantes de graduação.

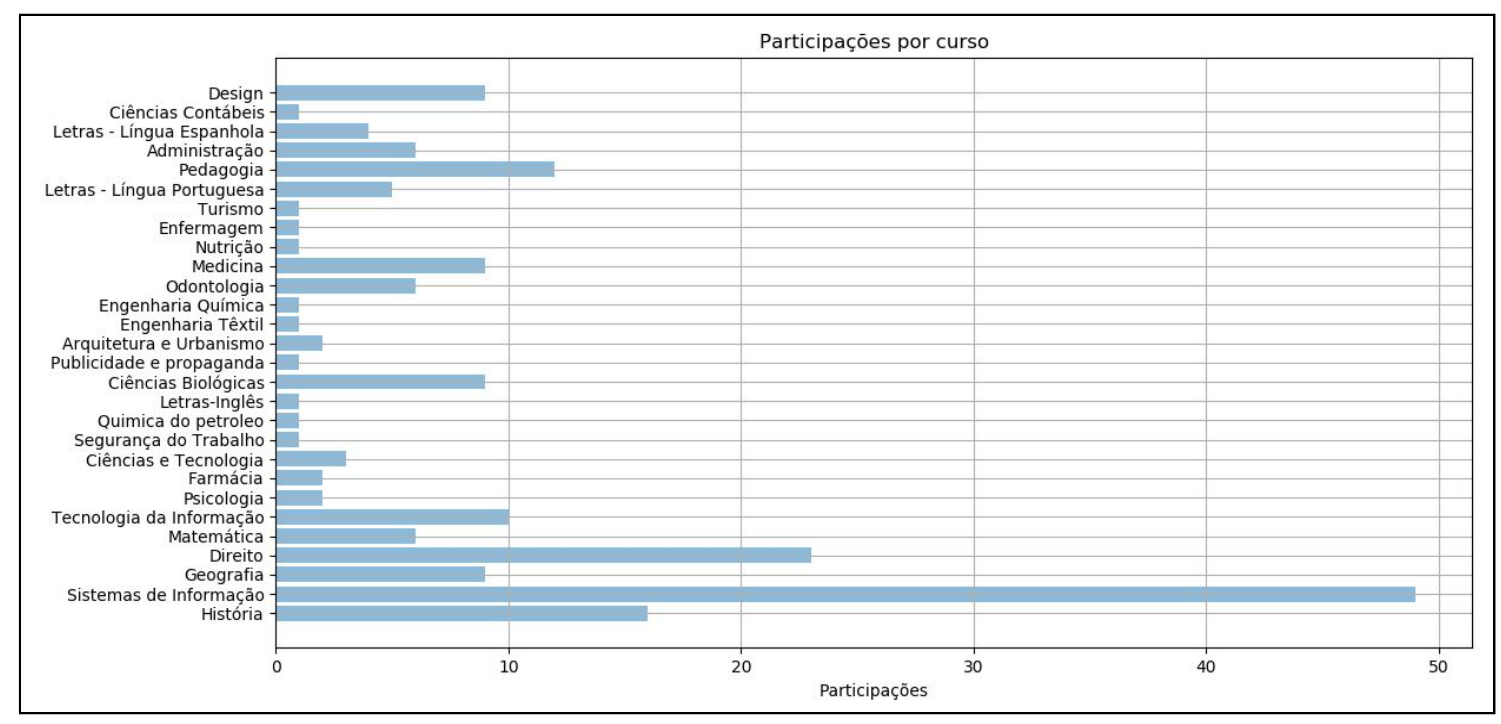

Figura 1: Participações por curso.

\subsection{Coleta de dados}

O processo de coleta dos dados dados se deu por meio de um questionário online com alternativas empregadas para estabelecer um nível de concordância ou discordância em relação a aspectos alusivos às expectativas e motivações para com o curso de graduação.

Além disso, as questões foram elaboradas com base na obra de Cavalcanti et al. (2014) e Ramos et al. (2014) e em acordo com a Escala de Likert, ou seja, elas apresentaram uma afirmação e, em seguida, ofereceram como opção de resposta uma escala com delimitações que contemplaram extremos - como "concordo totalmente" e "discordo totalmente". Ademais, foram incrementadas ao questionário perguntas de caráter subjetivo, três apenas para ter conhecimento do perfil do estudante, como por exemplo, curso de graduação, idade e período. E a quarta, com a finalidade de saber o fator que está influenciando o seu presente estado de motivação. 
VII Congresso Brasileiro de Informática na Educação (CBIE 2018)

Anais dos Workshops do VII Congresso Brasileiro de Informática na Educação (WCBIE 2018)

\subsection{Tratamento dos dados}

Para o tratamento da pergunta "Qual o principal motivo do seu atual estado de motivação?" foi utilizado a biblioteca NLTK (Natural Language Toolkit) da linguagem Python. Tal questionamento foi criado com a intenção de saber qual o principal motivo do estado motivacional do estudante em relação ao seu atual semestre.

A biblioteca foi empregada com propósito de eliminar de todas as respostas da pergunta anteriormente citada, alguns caracteres como sinais de de pontuação e números, que para o presente estudo não possuem relevância.

Já para o tratamento das questões que apresentaram alternativas de concordâncias, foi utilizado uma conversão da escala de Likert, a Figura 2 ilustra um caso.

\begin{tabular}{|c|c|c|c|c|}
\hline \multicolumn{5}{|c|}{ Estou motivado para cursar as disciplinas do semestre atual } \\
\hline Discordo totalmente & Discordo parcialmente & Indiferente & Condordo parcialmente & Concordo totalmente \\
\hline 1 & 2 & 3 & 4 & 5 \\
\hline
\end{tabular}

Figura 2: Exemplo da Escala de Likert.

Por exemplo, no presente estudo onde estava presente a afirmação "Discordo totalmente" houve a substituição pelo número 1 e onde encontrava-se "Concordo totalmente" houve a mudança por pelo número 5. Tal medida foi adotada tendo em vista que números são mais facilmente "entendidos" por gramas de computadores do que palavras.

\subsection{Análise de dados}

Para a análise dos dados, foi utilizada a linguagem Python. Pedregosa et al. (2011) afirma que a linguagem de programação Python vem se firmando como uma das linguagens mais prestigiadas para computação científica devido a sua natureza interativa e suas excelentes bibliotecas científicas, ou seja,é uma boa opção para análise exploratória de dados.

Ademais, a linguagem dispõe de uma sintaxe simples e objetiva que possibilita o programador se concentrar na resolução do problema e não em detalhes de implementação. Além disso, abarca um grande conjunto de bibliotecas destinadas a diversas áreas, desde computação científica até segurança. A seguir, são apresentadas as bibliotecas empregadas nesta pesquisa.

\subsubsection{Matplotlib}

O Matplotlib é uma biblioteca Python com recursos para a geração de gráficos $2 \mathrm{D}$ a partir de arrays. Tais gráficos podem ser criados com alta qualidade a partir de comandos simples e orientados com base em comandos gráficos do MATLAB (ambiente computacional numérico). Entre os cientistas de dados, essa ferramenta é bastante utilizada para visualização de dados através de gráficos de linha, barra, pizza, de dispersão, entre muitos outros. No presente trabalho, tal biblioteca com empregada para gerar o gráfico da Figura 1 presente na seção 5.1

\subsubsection{Pandas}

Outra biblioteca utilizada na análise dos dados foi a Pandas python por ser uma das ferramentas mais utilizadas por cientistas de dados para manipulação e análise de dados, ao lado do 
Matplotlib. Essa biblioteca possui estruturas rápidas e flexíveis criadas para tornar a análise de dados do mundo real mais fácil.

Essa biblioteca foi empregada, na presente obra, com o propósito de identificar possíveis regras de associação e classificação

\section{Resultados e Discussão}

Os frutos do experimento expõem sinais dos sentimentos dos universitários durante o início de um semestre e apontam possíveis causas de sua motivação, assim como informações a respeito de suas expectativas. Logo adiante os resultados são analisados.

\subsection{Motivação dos estudantes em relação ao atual semestre}

A partir da análise do grau de concordância dos estudantes à seguinte afirmação: "Estou motivado para cursar as disciplinas do semestre atual", foi obtido o resultado exibido na Figura 3.

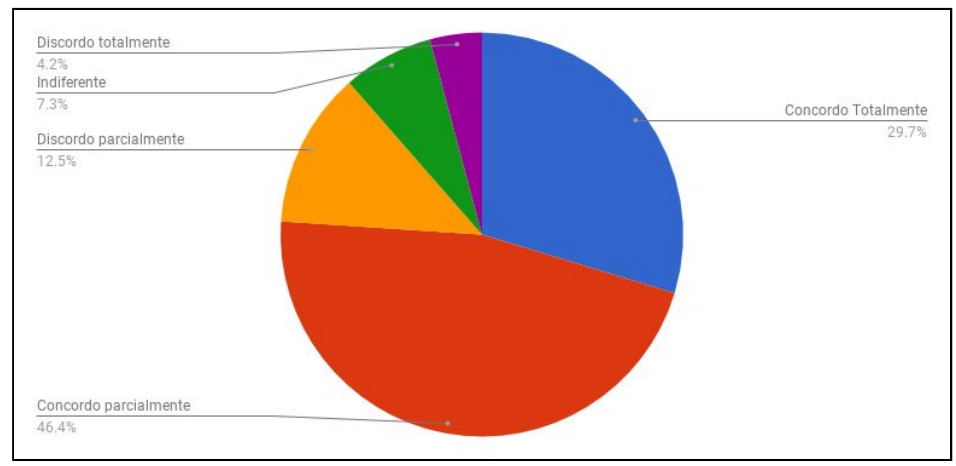

Figura 3: Motivação dos participantes.

A partir da observação da Figura 3, observa-se que a porcentagem de concordância (concordo parcialmente + concordo totalmente) em relação a afirmação anteriormente citada é de $76,1 \%$. Isso indica que a maioria dos participantes da pesquisa está determinada em cursar o atual período e que os outros 23,9\% (Indiferente + Discordo totalmente + Discordo parcialmente) não estão necessariamente motivados.

\begin{tabular}{|rr|}
\hline Costet da netodologia & Yédia de Motivaço \\
1 & 1.666667 \\
2 & 3.399909 \\
3 & 3.437509 \\
4 & 4.037383 \\
5 & 4.333333 \\
\hline
\end{tabular}

Figura 4: Relação entre metodologia e motivação

Já com base na Figura 4 percebe-se uma associação existente entre que a motivação dos estudantes e a metodologia de ensino proposta pelos professores. Para encontrar os valores expostos, foi realizado um média aritmética das respostas por grau de concordância em relação a seguinte afirmação: "Gostei da metodologia de ensino proposta pelos meus professores no atual semestre". 
Por meio da análise da figura anteriormente citada é possível afirmar que os discentes que deram uma avaliação positiva para a metodologia empregada pelos docentes, em média, apresentaram um estado motivacional proporcional a nota atribuída. Por exemplo, a média motivacional dos alunos que concordam totalmente (5) com a afirmação "Gostei da metodologia de ensino proposta pelos meus professores no atual semestre" é de 4.33, enquanto que a média dos que discordaram totalmente (1) é de aproximadamente 1.67.

Em outras palavras, o gosto pela metodologia apresentada pelos professores está diretamente relacionado com os estado motivacional dos estudantes.

\begin{tabular}{|rrr|}
\hline Formaçao antertor & Sexo & Mottvação média \\
Apenas ensino médio & Feminino & 3.839286 \\
Apenas enstino nédio & Masculino & 3.889597 \\
Apenas ensino nédio & Prefiro nao dizer & 4.060600 \\
Superior & Feminino & 3.846154 \\
Superior & Masculino & 4.083333 \\
Técnico & Feminino & 3.555556 \\
Técnico & Masculino & 4.062500 \\
\hline
\end{tabular}

Figura 5: Motivação por grupos

Em relação à Figura 5, através de uma análise de associação, ou seja, descoberta de grupos com características comuns, é possível observar a média de motivação por sexo e formação acadêmica dos voluntários da pesquisa. Através de sua análise constata-se que o grupo de indivíduos com a maior média motivacional, aproximadamente 4.083, é composta por homens que já possuem ensino superior, enquanto o grupo com a menor média, em torno de 3.556, é formado por pessoas do sexo feminino e que possuem apenas o ensino técnico.

Outra análise da Figura 5, diz respeito aos casos de formação acadêmica, em todos as ocorrências, as pessoas do sexo masculino apresentam uma média motivacional superior que as pessoas do sexo feminino.

Outro caso que deve ser analisado diz respeito a justificativa dada a seguinte pergunta: Qual o principal motivo do seu atual estado de motivação? As respostas foram processadas e estão expostas na Figura 6. Através da análise dela é possível verificar que as palavras mais usadas na justificativa foram Disciplina, Curso e Professor.

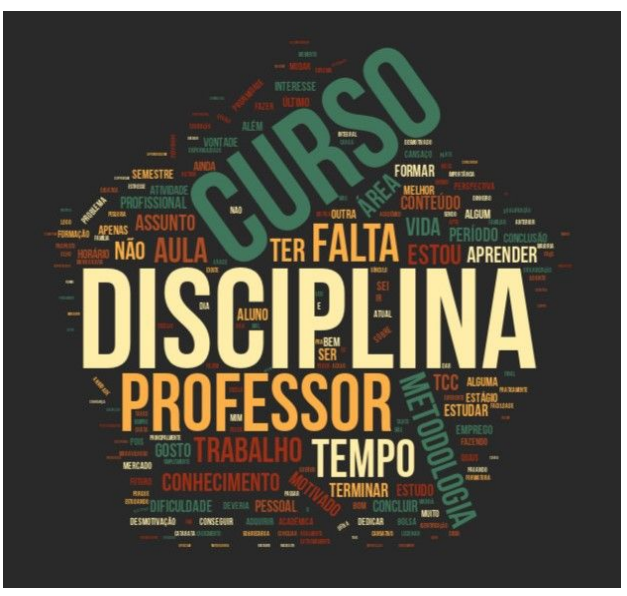

Figura 6: Nuvem de tags 
Ou seja, claramente os participantes desta pesquisa acreditam que sua principal fonte de motivação é externa e relacionada ao curso, em outras palavras, os professores e o corpo docente são o principais responsáveis pelo o incentivo dos estudantes a darem continuidade ao processo de aprendizagem no ambiente acadêmico.

\subsection{Expectativa dos estudantes em relação ao atual semestre}

Com base na análise do grau de concordância dos estudantes à seguinte afirmação: "Acredito que terei um bom relacionamento com os meus professores", foi obtido o resultado exposto na Figura 7.

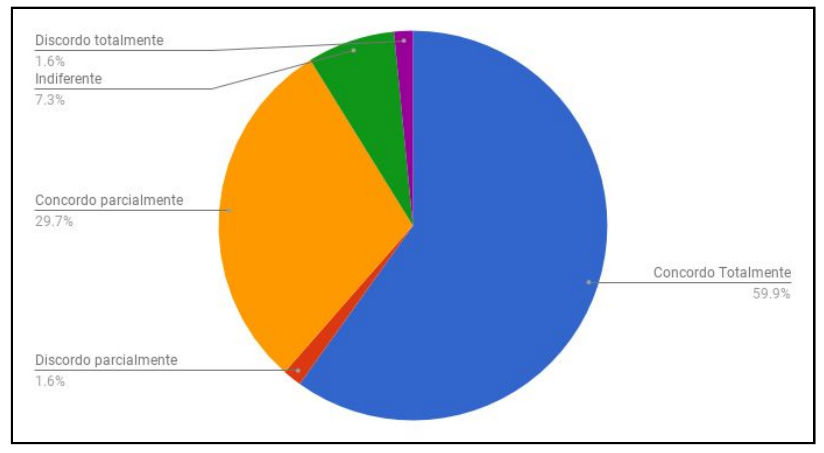

Figura 7: Bom relacionamento entre professor e estudante

Através da observação da Figura 7, nota-se que a porcentagem de concordância (concordo parcialmente + concordo totalmente) em relação a afirmação anteriormente citada é de $89,6 \%$. Isso indica que a maioria dos participantes da pesquisa possui grande expectativa em relação ao seus relacionamento com os seus professores e que os outros $10,4 \%$ (Indiferente + Discordo totalmente + Discordo parcialmente) não estão necessariamente esperam muito desse relacionamento.

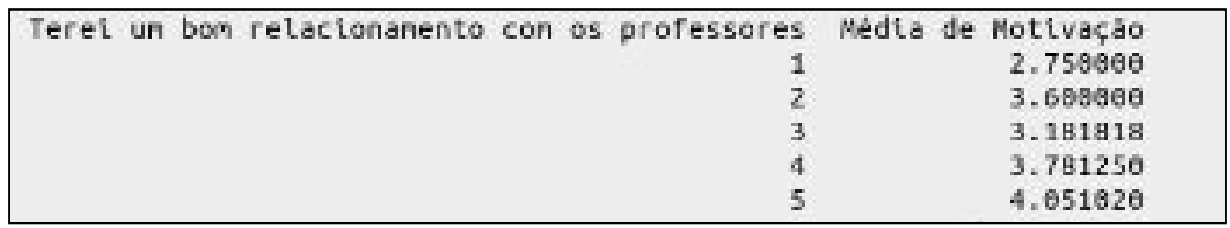

Figura 8: Relação entre relacionamento e motivação

Já de acordo com a Figura 8 evidencia-se que a expectativa criada pelos estudantes em relação ao seu futuro relacionamento com os docentes está associada a motivação, ou seja, em média, os discentes que acreditaram em um bom relacionamento apresentaram um estado motivacional mais elevado, enquanto os que desacreditaram no bom convívio apresentaram uma baixa média. 


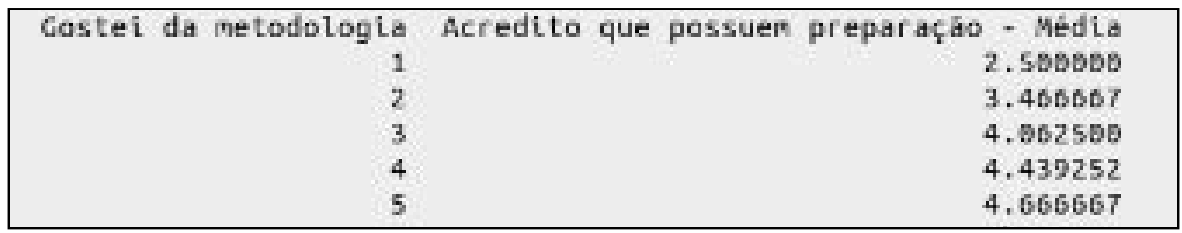

Figura 9: Relação entre metodologia e preparação dos docentes

E com base na análise da Figura 9, atenta-se a existência de uma relação direta entre a metodologia empregada pelos professores e a confiança que fulguram sob a ótica dos discentes. Em outras palavras, os voluntários que avaliaram positivamente a metodologia empregada pelos professores, em média, também deram maior credibilidade a sua preparação para lecionarem determinada grade curricular, por outro lado, os que classificaram negativamente não apresentam muita segurança a respeito da preparação do docente.

Isso indica que uma boa metodologia de ensino passa confiança acerca da preparação dos docentes para lecionarem as disciplinas que estão encarregados.

\section{Conclusões e trabalhos futuros}

Este artigo apresentou uma análise da viabilidade da concatenação de algumas tarefas da Mineração de dados, como associação e classificação, na condição de suporte para a construção de parâmetros relacionados à motivação e desapontamento de estudantes graduandos na modalidade de ensino presencial.

Os resultados apontaram bons níveis de motivação, porém ainda fortemente influenciada por fatores internos à universidade como as metodologias empregadas pelos professores em sala de aula. A mineração de dados educacionais pode revelar informações importantes para a tomada de decisão por professores e ou coordenadores de cursos com o intuito de aumentar a motivação dos estudantes a continuarem seus estudos. No presente trabalho foi empregado a linguagem Python, que se mostrou uma boa ferramenta para tratamento e análise de dados tendo em vista a sua facilidade de uso e existência de bibliotecas que dão suporte a mineração de dados.

Recomenda-se como trabalho futuro a mineração de texto com foco na análise de sentimentos em perguntas discursivas, com o objetivo de detectar padrões sentimentais entre estudantes de graduação, em relação a satisfação perante o emprego de metodologias e conteúdo abordados em cursos presenciais.

\section{Referências}

AIRES et al. (2017). Mineração de dados educacionais para análise preditiva da evasão escolar. Disponível em:<http://www.enangrad.org.br/2017/pdf/2017 ENANGRAD176.pdf>. Acesso em: 06 de jun. 2018.

BAKER, Ryan; ISOTANI, Seiji; CARVALHO, Adriana. Mineração de dados educacionais: Oportunidades para o brasil. Brazilian Journal of Computers in Education, v. 19, n. 02, p. 03, 2011. 
VII Congresso Brasileiro de Informática na Educação (CBIE 2018)

Anais dos Workshops do VII Congresso Brasileiro de Informática na Educação (WCBIE 2018)

BRAMER, Max. Principles of Data Mining. London: Springer, 2007.

CAMILO, Cássio Oliveira; SILVA, João Carlos da. Mineração de dados: Conceitos, tarefas, métodos e ferramentas. Universidade Federal de Goiás (UFC), p. 1-29, 2009.

CAVALCANTI, A. G. G. et al. Mineração e Visualização de Dados Educacionais: identificação de fatores que afetam a motivação de alunos na educação a distância. XIV Escola Regional de Computação Bahia, Alagoas e Sergipe (ERBASE), 2014.

CAVENAGHI, Ana Raquel Abelha; BZUNECK, José Aloyseo. A motivação de alunos adolescentes enquanto desafio na formação do professor. In: CONGRESSO NACIONAL DE EDUCAÇÃO. 2009. p. 1478-1489.

COSTA, Andrea, Técnicas de coleta de dados e instrumentos de pesquisa. 2016.

GOUVEIA, Luis Borges. O elearning para suporte ao ensino presencial universitário. SOPCOM: Associação Portuguesa de Ciências da Comunicação, p. 1559-1571, 2005.

MANZATO, Antonio José; SANTOS, Adriana Barbosa. A elaboração de questionários na pesquisa quantitativa.Departamento de Ciência de Computação e Estatística-IBILCE-UNESP pp. 1-17. 2012.

OLSON, David L.; DELEN, Dursun. Advanced data mining techniques. Springer Science \& Business Media, 2008.

PEDREGOSA, Fabian et al. Scikit-learn: Machine learning in Python. Journal of machine learning research, v. 12, n. Oct, p. 2825-2830, 2011.

RAMOS, Jorge Luis Cavalcanti et al. Análise de expectativas e habilidades discentes na modalidade de ensino a distância. In: Actas de la $9^{\mathbf{a}}$ Conferencia Ibérica de Sistemas y Tecnologías de Informacion. Barcelona-Espanha. 2014. p. 131-136.

SANTOS, Fabio; SILVEIRA, Ismar Frango; LECHUGO, Carla. Mineração da percepção do aluno: Mineração de Dados Educacionais na geração de indicadores para a avaliação de práticas pedagógicas docentes. In: Anais dos Workshops do Congresso Brasileiro de Informática na Educação. 2016. p. 1473.

SANTOS, Rafael et al. Conceitos de Mineração de Dados na Web. XV Simpósio Brasileiro de Sistemas Multimídia e Web, VI Simpósio Brasileiro de Sistemas Colaborativos-Anais, MM Teixeira, CAC Teixeira, FAM Trinta, e P. PM Farias, Eds, p. 81-124, 2009.

SCHEUER, Oliver; MCLAREN, Bruce M. Educational data mining. Springer, pp. 1075-1079. 2012.

SUMATHI, S; SIVANANDAM, SN. Introduction to data mining principles. 1-20. 2006. 